\title{
Research Paper: The Measurement of Height Via 3D Scans of the Seventh Cervical Vertebra in Iranian Adults
}

\author{
Seyed Reza Saadat Mostafavi ${ }^{1}$ Q, Azadeh Memarian ${ }^{2 *}$ Omid Motamedi ${ }^{1}$, Arash Heidari
}

1. Department of Radiology, Faculty of Medicine, Iran University of Medical Sciences, Tehran, Iran.

2. Department of Forensic \& Legal Medicine, Faculty of Medicine, Iran University of Medical Sciences, Tehran, Iran.

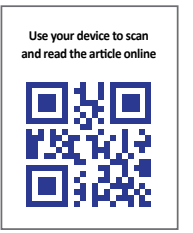

Citation: Mostafavi SRS, Memarian A. The Measurement of Height Via 3D Scans of the Seventh Cervical Vertebra in Iranian Adults. International Journal of Medical Toxicology and Forensic Medicine. 2020; 10(2):27887. https://doi.org/10.32598/ ijmtfm.v10i2.27887

https://doi.org/10.32598/ijmtfm.v10i2.27887

\section{(c) $0(3)$}

Article info:

Received: 24 Nov 2019

First Revision: 10 Dec 2019

Accepted: 08 Jan 2020

Published: 28 Jun 2020

\section{Keywords:}

Height, The seventh cervical vertebrae, CT scan

\section{ABSTRACT}

Background: The cervical vertebrae are more durable than other skeletal components, and maybe the only remains of a deceased, and their role in determining the height of the deceased has been underestimated. The present study investigated the role of linear differential dimensions of the seventh cervical vertebrae in determining the height of the Iranian adult population using CT scans.

Methods: In this cross-sectional study, height were evaluated by 10 indices of the seventh cervical vertebra. The indices were obtained through a CT scan of 66 adult patients, $\geq 18$ years of age, who referred to Rasoul Akram and Firoozgar hospitals for spine CT scan. The sampling method was random, and the study was performed during the first six months of 2018.

Results: Four indices of the Length of the Inferior Facets) LIF, (Length of the Inferior Surface of the Vertebral Body) LIVB, (Width of the Inf surface of the Vertebral Body) WIVB, (Length of Spinous Process ) LSP. were statistically significant differences.

Conclusion: The results of this study show the accuracy of linear dimensions of the seventh cervical vertebrae for determining height from skeletal remains in the Iranian adult population.

\section{Introduction}

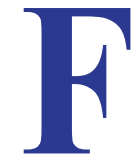

orensic physicians should examine many decayed corpses that their identification is sometimes difficult. Such cases require a specific approach, as the remains of the skeleton are the only clue for their identification [1]. The estimation of age and determination of gender, height, and race are the first parameters of identification [2]. In this regard, skeletons and teeth are more resis- tant to decay and play a significant role in identifying the corpses. Considering the relationship between the height and morphology of spine vertebrae and in cases in which just a segment of the spine is available, the morphology of spine vertebrae can help us discover different skeletal features, including stature [3].

The skeletal features of individuals are characterized by different cervical, thoracic, and lumbar spines dimensions. To measure the durability of the cervical spine, all the de-

* Corresponding Author:

Azadeh Memarian, MD.

Address: Department of Forensic \& Legal Medicine, Faculty of Medicine, Iran University of Medical Sciences, Tehran, Iran.

Tel: +98 (21) 66551201

E-mail:memarian.a@iums.ac.ir 
tails of soft and hard tissues and the brittle elements of the vertebra, as well as metric indices of spine bones, must be investigated. Also, the evaluation of the cross-sectional surface of the vertebral body and its middle cavity is essential for the comprehensive investigation of the vertebra. Thus, each part of the spine, including the cervical area, can help us to find the height in cases with severe trauma [4].

Different studies have tried to set up standards for the identification of corpses from the remains. Their modeling was based on the dimensions and morphology of skeletons in this regard. However, there are still limitations to the identification based on the skeletal remains. In the majority of cases, the skeletal remains are not unified and have segregated or deformed during the process of decay [5]. Furthermore, most of the studies on modeling and presenting facts and figures for the identification of skeletons have focused on specific populations that cannot be used in other tribal, racial, or regional groups. As the differences in the height of skeletons are affected by the genetic and environmental factors, reviewing the anthropological features and using it in the identification of corpses are applied regionally. Thus, it is not possible to generalize the studies on a specific sample to other peoples due to the biological features of individuals $[2,6]$.

The present study aimed to enhance the accuracy of identification by evaluating the height based on the three-dimensional CT scans of the seventh cervical spine in Iranian adults.

\section{Materials and Methods}

This research is a cross-sectional study. In this study, 66 individuals ( 33 males and 33 females) aged $\geq 18$ years who underwent Cervical Vertebrae CT scan in Rasoul Akram and Firoozgar hospitals were selected randomly. A randomized sampling method was used, and all participants signed informed consent.

The sample size was determined according to Vasavada study in 2008. By considering a confidence interval of 0.05 and the study power of $90 \%$, the required sample size for this study was 33 for male and 33 for female:

$$
n_{1}=n_{2}=\frac{\left(S_{1}{ }^{2} \pm S_{2}{ }^{2}\right)\left(Z_{1-\alpha / 2} \pm Z_{1-\beta}\right)^{2}}{\left(\bar{X}_{1} \pm \bar{X}_{2}\right)^{2}}
$$

$X^{1}$ (The mean height of the seventh cervical vertebra in Men) $=24.6$

\section{$X^{2}$ (The mean height of the seventh cervical vertebra in Women) $=22.6$}

$S^{l}(S D$ in Men $)=2.7$

$S^{2}(S D$ in Women $)=2.4$

$Z_{1-\alpha / 2}=1.96 ; Z_{1-\beta}=1.29$;

$n 1=n 2=33 n 1=n 2=33$

S: alpha and beta are reffered to standard deviation of type I and II of error.

$\mathrm{Z}$ : is an standardized value of type one error. For calculating sample size we consider these indexes when we use comparing two mean formula.

$\mathrm{N}_{1}=\mathrm{N}_{2}=33$ : The sample size required is 33 for men and 33 for women

Sup:Superior

Inf: Inferior

The exclusion criteria were as follows: age under 18 years and history of congenital anomalies in the cervical spine, trauma to the neck with any severity, or any structural, metabolic, or rheumatologic disorders in the cervical spine, or any surgical or therapeutic intervention in the neck area.

The linear dimensions of the seventh cervical vertebrae were measured using three dimensional CT scan (Slice, Toshiba, Japan) with Multiplanar Reconstruction (MPR) and volume rendering at sagittal and horizontal sections. The thickness of the slices was $1 \mathrm{~mm}$. Ten indices of the seventh cervical vertebrae were measured: length of the Superior Facets (LSF), the Width of the Superior Facets, (WSF), Length of the Inferior Facets, (LIF) (Figure 1), the Width of the Inferior Facets (WIF), Length of the Vertebral Foramen (LVF), the Wdth of the Vertebral Foramen (WVF), Length of the Inferior Surface of the Vertebral Body, (LIVB) (Figure 2), the Width of the Inferior Surface of the Vertebral Body (WIVB) (Figure 3), Length of the Spinous Process (LSP) (Figure 4), and Height of the Spinous Process (HSP).

\section{Statistical analysis}

The obtained data were analyzed in SPSS v. 23. For quantitative variables, t-test was used to compare the data, and the Pearson correlation test was used to investigate the relationship between height and second cervical vertebrae indexes. The results were significant at $\mathrm{P}<0.05$. The Kolmogorov-Smirnov test (K-S) test was used to determine the normal distribution of data. 


\section{Results}

The present study was done on 66 adults volunteering for a cervical CT scan, of which 33 females and 33 males in the 18-83 age range participated. The results from the age distribution in genders suggest no statistically significant difference between females and males $(\mathrm{P}=0.938)$, and the age of both sexes was the same. Besides, in the present study, ten indices from the seventh cervical vertebra were investigated. Table 1 presents the indices of each vertebra in the whole population.

To investigate the relationship between the age and the indices of the seventh vertebra, we used the Pearson correlation test for both genders (depending on the normality of distribution). Based on the results, WIF and HSP indices had a weak and positive relationship with age. Also, LSF had a weak and positive relationship with age, and LIVB and WIVB indices had a potential and positive correlation with age. To investigate the relationship between the height and the indices of the seventh vertebra, we used the Pearson correlation test for both genders (depending on the normality of distribution) (Table 2).

According to Table 2, the LIF index had a weak and significant positive correlation with height and LIVB, WIVB and LSP indices had a potential and significant positive relationship with height.

Figure 1 shows the significant correlation of the height with LIF $(\mathrm{P}<0.012)$. Figure 2 displays the significant relationship of the height with LIVB $(\mathrm{P}<0.010)$. Figure 3 shows the significant correlation of the height with WIVB $(\mathrm{P}<0.0001)$. Figure 4 shows the significant correlation of the height with Lsp $(\mathrm{P}<0.002)$. Based on the results, there is a significant relationship between height and variables of LSP, WIVB, LIVB, LIF.

\section{Discussion}

The present study assessed the relationships of the seventh cervical vertebra indices with the height of Iranian adults, considering the cervical ST scan indices. The results from this study suggested a significant relationship between four (LIF, LIVB, WIVB, LSP) (of ten) indices of the seventh cervical vertebra and height. According to the study of Suguru Torimitsu et al. on the Japanese population, there is an important and significant relationship between the DA, AS, DS vertebrae, and height, which is consistent with the results of the present study [7]. Moreover, K.R. Nagesh study on the Indian population confirmed this relationship [1]. In the present study, despite the relationship between four indices of LIF, LIVB, WIVB, and LSP with height, the standard error was high.

Although these indices can be a powerful tool for estimating the height, this calculation is reliable when the relationship between other cervical vertebrae, even other spines, and tall bones are investigated and used for the estimation of height. As it was observed in the age range of the 69 years, the possibility of degenerative changes affecting the vertebrae must be considered, too. Other studies showed the relationship between height and cervical vertebrae dimensions $[1,8]$.

Table 1. Description of indices of the seventh cervical vertebra in the whole population

\begin{tabular}{|c|c|c|c|}
\hline Indices & Mean $\pm S D$ & Min & Max \\
\hline LSF & $9.09 \pm 1.189$ & 7 & 12 \\
\hline WSF & $12.13 \pm 1.215$ & 9 & 15 \\
\hline LIF & $9.57 \pm 1.199$ & 7 & 13 \\
\hline WIF & $12.17 \pm 1.503$ & 8 & 15 \\
\hline LVF & $15.27 \pm 1.963$ & 13 & 24 \\
\hline WVF & $24.79 \pm 1.849$ & 22 & 30 \\
\hline LIVB & $16.33 \pm 1.878$ & 12 & 21 \\
\hline WIVB & $25.81 \pm 2.555$ & 21 & 33 \\
\hline LSP & $24 \pm 3.985$ & 14 & 33 \\
\hline HSP & $7.76 \pm 1.419$ & 5 & 11 \\
\hline
\end{tabular}


Table 2. The correlation between the height and indices of the seventh vertebra in the whole population

\begin{tabular}{ccc}
\hline Indices & Correlation Coefficient & $\mathbf{P}$ \\
\hline LSF & 0.221 & 0.065 \\
WSF & 0.162 & 0.180 \\
\hline LIF & 0.299 & 0.012 \\
\hline WIF & 0.169 & 0.162 \\
\hline LVF & 0.105 & 0.388 \\
\hline WVF & 0.109 & 0.368 \\
\hline LIVB & 0.306 & 0.010 \\
WIVB & 0.449 & 0.0001 \\
\hline LSP & 0.371 & 0.002 \\
\hline HSP & 0.066 & 0.585 \\
\hline
\end{tabular}

The cervical vertebrae parameters were identified as an accurate criterion for estimating the age of bone [9]. Another study of K.R. Nagesh et al. introduced height as a reliable parameter for the identification of an individual's identity. The lengths of different segments of the vertebral column were measured along with the anterior surface in 72 males and 45 females. The present study suggested that the estimation of height from various segments of the spine, especially cervical vertebrae, is possible and can be helpful when the corpses are segregated, burnt, or injured severely, or the tall bones are not available [1].

Mildred Trotter et al. confirmed the above study results [10]. Also, another study of Mildred Trotter et al. on tall bones and cervical vertebrae of corpses remained from the second world war suggested that dimensions and sizes of bones can be used for estimation of the height and pheno-

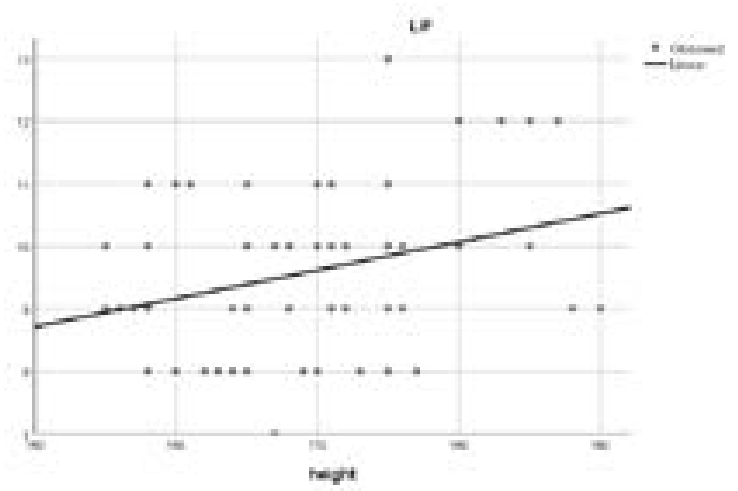

International Journal of
Medical Toxicology \& forensic Medicine

Figure 1. Correlation of height with LIF type features [8, 11]. CP Campobasso et al. suggested a direct relationship between scapular bone dimensions and height [12]. Mubarak Ariyo Bidmos et al. conducted a study on indigenous South Africans and collected a total of 100 full skeletons from Raymond A. collection with the same gender distribution. The height of each skeleton was estimated using Fully's method [13]. The present study confirmed the helpfulness of skeletal segments of indigenous South Africans for the estimation of height [14].

Wu RQ et al. conducted a study on Yangtze Delta and analyzed 993 remains [15]. They compared the dimensions and indices of the cervical vertebrae to discover its relationship with height. The results showed that the forensic physicians could use the dimensions and size of cervical bones as a useful, easy, and fast method for accurate estimation of height.

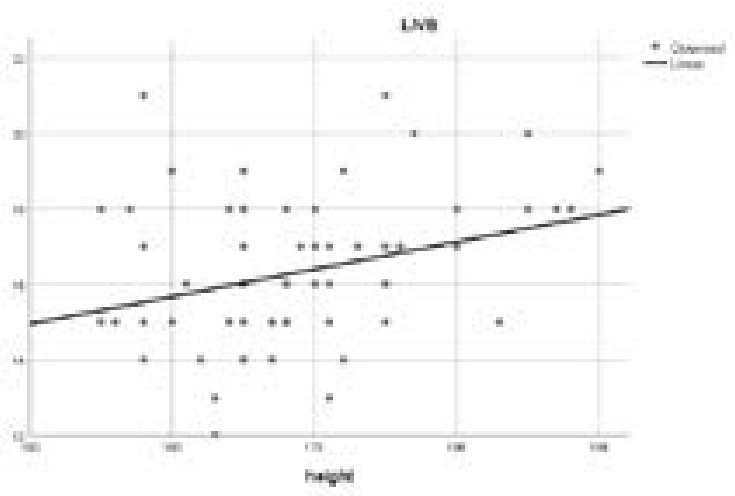

International Journal of
Medical Toxicology \& forensic Medicine

Figure 2. Correlation of height with LIVB 


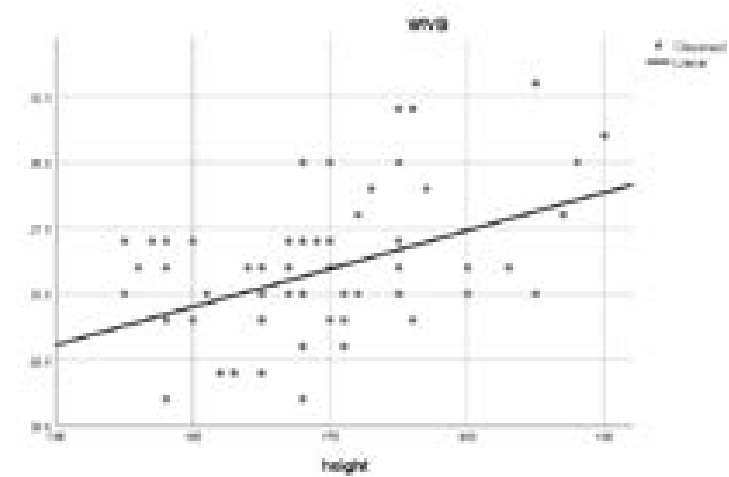

International Journal of
Medical Toxicology \& forensic Medicin

Figure 3. The correlation of height with WIVB

According to Rodríguez $\mathrm{S}$ et al., although femur and tibia are ideal for estimating the height and other anthropometric indices, smaller bones such as cervical vertebrae (the first and second ones) could be helpful, too [6]. The results of their study on 203 Spanish people showed a significant relationship between the size of the second cervical vertebra and height.

Considering different dimensions in the mentioned cases, the estimation of height was considerably accurate based on the comparison of the present study with studies on indices of the seventh cervical vertebra. As the present study used the Pearson correlation tests due to the normality of data distribution, it confirmed the results of the previous studies. Although the dispersion of studies on the estimation of height in determining the dimensions of the seventh cervical vertebra made the comparison of the results impossible, generally, the results suggested the relative accuracy of the dimensions of this vertebra.

The identification of corpses by different segments of the skeleton has already been investigated in many studies. For instance, the skeletal features are detected by the variety of proximal ulna morphology $[16,17]$. Furthermore, it is possible to determine nutritional status from the skeleton, and the length of limbs can indicate the height $[18,19]$.

The difference in body sizes can be defined as a confounder for the relationship between the vertebra size and height. Because of the limitations in the present study, it was not possible to measure the dimensions of the body. Thus, matching the dimensions for the removal of confounder and finding independent variables for determining the height from the vertebrae dimensions are suggested for future studies. Moreover, committing errors is probable in the measurement of dimensions. Still, as it was done by software, its probability of bias or measurement error is less than that of manual measurements. Because a CT scan is an

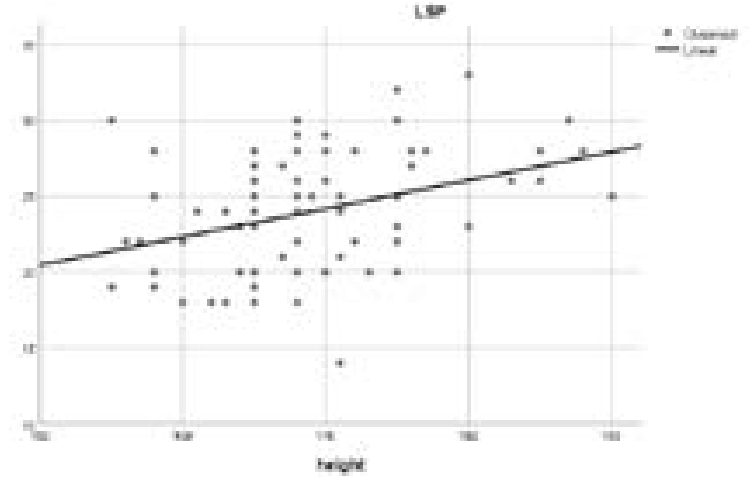

International Journal of
Medical Toxicology \& Forensic Medicine

Figure 4. The correlation of height with LSP

illustration regenerator, it differs from the real dimensions though these differences are subtle and must be considered for the interpretation.

\section{Conclusion}

In this study four indices of the (length of the Inferior Facets) LIF, (length of the Inferior Surface of the vertebral body) LIVB, (width of the inf surface of the vertebral body) WIVB, (length of spinous process) LSP via 3D scan were statistically significant differences. The results of this study suggested the accuracy of some $7^{\text {th }}$ cervical vertebrae dimensions to estimate the height, Therefore, 3D scanning can be a good alternative to autopsy in evaluating vertebral parameters. Thus, it is recommended to use it for the identification in Iranian forensic medicine. To determine the standards, we need to conduct studies with a large sample size and controlling confounders.

\section{Ethical Considerations}

\section{Compliance with ethical guidelines}

All ethical principles were considered in this article.

\section{Funding}

This research did not receive any specific grant from funding agencies in the public, commercial, or not-forprofit sectors.

\section{Author's contributions}

All authors contributed in designing, running, and writing all parts of the article. 


\section{Conflict of interest}

The authors declared no conflict of interest with respect to this manuscript.

\section{Acknowledgements}

The Authors would thanks Professor Aghakhani for the guidence of this research.

\section{References}

[1] Nagesh K, Kumar GP. Estimation of stature from vertebral column length in South Indians. Leg Med (Tokyo). 2006; 8(5):269-72. [DOI:10.1016/j.legalmed.2006.05.007] [PMID]

[2] Gilsanz V, Boechat MI, Gilsanz R, Loro ML, Roe TF, Goodman WG. Gender differences in vertebral sizes in adults: Biomechanical implications. Radiology. 1994; 190(3):678-82. [DOI:10.1148/ radiology.190.3.8115609] [PMID]

[3] Gilsanz V, Kovanlikaya A, Costin G, Roe TF, Sayre J, Kaufman F. Differential effect of gender on the sizes of the bones in the axial and appendicular skeletons. J Clin Endocrinol Metab. 1997; 82(5):1603-7. [DOI:10.1210/jcem.82.5.3942]

[4] Mosekilde L, Mosekilde L. Sex differences in age-related changes in vertebral body size, density and biomechanical competence in normal individuals. Bone. 1990; 11(2):67-73. [DOI:10.1016/87563282(90)90052-Z]

[5] Bidmos MA, Gibbon VE, Štrkalj G. Recent advances in sex identification of human skeletal remains in South Africa. S Afr J Sci. 2010; 106(11-12):1-6. [DOI:10.4102/sajs.v106i11/12.238]

[6] Rodríguez S, Rodríguez-Calvo MS, González A, Febrero-Bande M, Muñoz-Barús JI. Estimating height from the first and second cervical vertebrae in a Spanish population. Leg Med. 2016; 19:8892. [DOI:10.1016/j.legalmed.2015.08.002] [PMID]

[7] Torimitsu S, Makino Y, Saitoh H, Sakuma A, Ishii N, Hayakawa $\mathrm{M}$, et al. Stature estimation in Japanese cadavers based on the second cervical vertebra measured using multidetector computed tomography. Leg Med. 2015; 17(3):145-9. [DOI:10.1016/j. legalmed.2014.11.003] [PMID]

[8] Trotter M, Gleser GC. A re-evaluation of estimation of stature based on measurements of stature taken during life and of long bones after death. Am J Phys Anthropol. 1958; 16(1):79-123. [DOI:10.1002/ajpa.1330430112] [PMID]

[9] Mito T, Sato K, Mitani H. Cervical vertebral bone age in girls. Am J Orthod Dentofacial Orthop. 2002; 122(4):380-5. [DOI:10.1067/ mod.2002.126896] [PMID]

[10] Trotter M, Gleser GC. Estimation of stature from long bones of American Whites and Negroes. Am J Phys Anthropol. 1952; 10(4):463-514. [DOI:10.1002/ajpa.1330100407] [PMID]

[11] Trotter M. A re-examination of estimation of stature based on measurements of stature taken during life and of long bones after death. Am J Phys Anthropol. 1958; 10:463-514. [DOI:10.1002/ ajpa.1330100407] [PMID]
[12] Campobasso CP, Di Vella G, Introna F. Using scapular measurements in regression formulae for the estimation of stature. Boll Soc Ital Biol Sper. 1998; 74(7-8):75-82. [PMID]

[13] Bidmos M, Asala S. Calcaneal measurement in estimation of stature of South African blacks. Am J Phys Anthropol. 2005; 126(3):335-42. [DOI:10.1002/ajpa.20063] [PMID]

[14] Bidmos MA. Estimation of stature using fragmentary femora in indigenous South Africans. Int J Legal Med. 2008; 122(4):293-9. [DOI:10.1007/s00414-007-0206-2] [PMID]

[15] Wu RQ, Wang T, Shi Q, Xiao B, Ma KJ, Chen X. [Adult stature estimation by multiple parameters of body torso segment (Chinese)]. Fa yi xue za zhi. 2017; 33(3):236-8. [DOI:10.3969/j. issn.1004-5619.2017.03.004] [PMID]

[16] Bruzek J, Murail P. Methodology and reliability of sex determination from the skeleton. In: Forensic anthropology and medicine. Berlin: Springer; 2006. [DOI:10.1007/978-1-59745-099-79]

[17] Cowal LS, Pastor RF. Dimensional variation in the proximal ulna: evaluation of a metric method for sex assessment. Am J Phys Anthropol. 2008; 135(4):469-78. [DOI:10.1002/ajpa.20771] [PMID]

[18] Ulijaszek SJ, Kerr DA. Anthropometric measurement error and the assessment of nutritional status. Br J Nutr. 1999; 82(3):165-77. [DOI:10.1017/S0007114599001348] [PMID]

[19] Case DT, Ross AH. Sex determination from hand and foot bone lengths. J Forensic Sci. 2007; 52(2):264-70. [DOI:10.1111/ j.1556-4029.2006.00365.x] [PMID] 